\title{
FORMAL SPACES WITH FINITE-DIMENSIONAL RATIONAL HOMOTOPY
}

BY

\author{
YVES FELIX AND STEPHEN HALPERIN
}

\begin{abstract}
Let $S$ be a simply connected space. There is a certain principal fibration $K_{1} \rightarrow E \stackrel{\pi}{\rightarrow} K_{0}$ in which $K_{1}$ and $K_{0}$ are products of rational Eilenberg-Mac Lane spaces and a continuous map $\phi: S \rightarrow E$ such that in particular $\phi_{0}=\pi \circ \phi$ maps the primitive rational homology of $S$ isomorphically to that of $K_{0}$. A main result of this paper is the

THEOREM. If $\operatorname{dim} \pi_{*}(S) \otimes \mathbf{Q}<\infty$ then $\phi$ is a rational homotopy equivalence if and only if all the primitive homology in $H_{*}(S ; \mathbf{Q})$ and $H_{*}\left(K_{0}, S ; \mathbf{Q}\right.$ ) can (up to integral multiples) be represented by spheres and disk-sphere pairs.

COROLLARY. If $S$ is formal, $\phi$ is a rational homotopy equivalence.
\end{abstract}

1. Introduction. Let $S$ be a simply connected space with $\operatorname{dim} H_{p}(S)<\infty$ for all $p$. ( $H_{*}(S)$ denotes singular cohomology with rational coefficients.) Let $P_{*}(S) \subset H_{*}(S)$ denote the primitive subspace of the coalgebra $H_{*}(S)$, and fix a homogeneous basis $\alpha_{i} \in P_{m_{i}}(S)$.

Set $K_{0}=\Pi_{i} K\left(\mathbf{Q} ; m_{i}\right)$ and let $\beta_{j} \in H^{m_{j}}\left(K_{0}\right)$ be the image of the fundamental class of $K\left(\mathbf{Q} ; m_{j}\right)$ in $K_{0}$. Choose a continuous map $\phi_{0}: S \rightarrow K_{0}$ so that $\left\langle\left(\phi_{0}\right)_{*} \alpha_{i}, \beta_{j}\right\rangle$ $=\delta_{i j}$; then $\left(\phi_{0}\right)_{*}: P_{*}(S) \stackrel{\cong}{\rightarrow} P_{*}\left(K_{0}\right)$. The relative homology $H_{*}\left(K_{0}, S\right)$ is a comodule over $H_{*}\left(K_{0}\right)$; let $P_{*}\left(K_{0}, S\right)$ denote the primitive subspace with homogeneous basis $\gamma_{i} \in P_{n_{i}}\left(K_{0}, S\right)$.

Because the $\phi_{0}^{*} \beta_{j}$ are dual to $P_{*}(S)$ they generate the algebra $H^{*}(S)$, and so $\phi_{0}^{*}$ is surjective. We may thus interpret $H^{*}\left(K_{0}, S\right)$ as an ideal in $H^{*}\left(K_{0}\right)$. Set $K_{1}=$ $\Pi_{i} K\left(\mathbf{Q} ; n_{i}-1\right)$ and let

$$
K_{1} \rightarrow E \rightarrow K_{0}
$$

be a principal fibration such that if $\omega_{i} \in H^{n_{i}}\left(K_{0}\right)$ is the transgressed fundamental class of $K\left(\mathbf{Q} ; n_{i}-1\right)$ then $\omega_{i} \in H^{n_{i}}\left(K_{0}, S\right)$ and $\left\langle\gamma_{i}, \omega_{j}\right\rangle=\delta_{i j}$. Standard obstruction theory shows that $\phi_{0}$ lifts to a continuous map $\phi_{1}: S \rightarrow E$.

Call classes $\alpha \in H_{p}(S), \beta \in H_{p}(K, S)$ spherical if some integral multiple of $\alpha$ (respectively, $\beta$ ) can be represented by $S^{p}$ (respectively, by $\left(D^{p}, S^{p-1}\right)$ ). Spherical

Received by the editors June 16, 1980.

1980 Mathematics Subject Classification. Primary 55P62, 55Q52.

Key words and phrases. Rational homotopy, minimal model, formality, Eilenberg-Moore spectral sequence. 
homology is always primitive, but the reverse inclusion usually fails. Indeed, a main theorem of this paper reads

THEOREM I. Suppose $S$ is a simply connected space such that $\operatorname{dim} \pi_{*}(S) \otimes \mathbf{Q}<\infty$. Then (with the notation above) the following two conditions are equivalent:

(1) the primitive classes in $H_{*}(S)$ and $H_{*}\left(K_{0}, S\right)$ are all spherical,

(2) the continuous map $\phi_{1}: S \rightarrow E$ is a rational homotopy equivalence.

Moreover, when they hold, the integers $n_{i}$ are all even; the classes $\omega_{i}$ form a prime sequence in the free commutative graded algebra $H^{*}\left(K_{0}\right)$; and $H^{*}(S) \cong H^{*}\left(K_{0}\right) / I$, where $I$ is the ideal generated by the $\omega_{i}$.

REMARK. A prime (or regular) sequence in an algebra $H$ is a sequence $\omega_{1}, \ldots$ such that in the factor algebra obtained by setting $\omega_{1}=\cdots=\omega_{i-1}=0$, the image of $\omega_{i}$ is not a zero divisor $(i=1,2, \ldots)$.

Theorem I can be restated in an apparently very different form. Recall that the Eilenberg-Moore spectral sequence for $S$ [9] is a 2nd quadrant spectral sequence, converging to $H^{*}(\Omega S)$, which is a stronger invariant than the algebra $H^{*}(S)$. (Indeed $d_{1}: E_{1}^{-2, *} \rightarrow E_{1}^{-1, *}$ is simply the map $H^{+}(S) \otimes H^{+}(S) \rightarrow H^{+}(S)$.) The higher differentials are a further (but still incomplete-cf. [7, §8.13]) invariant of the rational homotopy type of $S$.

For certain spaces however (called formal spaces - the precise definition is given below) the rational homotopy type is a formal consequence of the cohomology algebra. Thus two formal spaces with isomorphic cohomology algebras have the same rational homotopy type. If a simply connected commutative graded algebra $H$ over $\mathrm{Q}$ has the property that $H(S) \cong H \Rightarrow S$ is formal, then $H$ is called intrinsically formal.

An algebra of the form

$$
\wedge X=\text { exterior algebra }\left(X^{\text {odd }}\right) \otimes \text { symmetric algebra }\left(X^{\text {even }}\right)
$$

is intrinsically formal. (Such algebras are exactly the cohomology algebras for a product of $K(\mathbf{Q} ; n)$ 's, $n$ possibly varying.) More generally if $H$ is the quotient of $\wedge X$ by an ideal generated by a prime sequence then $H$ is intrinsically formal (cf. Remark 3.1). We call algebras of this form hyperformal. Since a wedge of odd spheres is intrinsically formal [7, Theorem 1.5] but usually not hyperformal, none of the implications

$$
H(S) \text { hyperformal } \Rightarrow H(S) \text { intrinsically formal } \Rightarrow S \text { formal }
$$

can be reversed.

On the other hand the Eilenberg-Moore spectral sequence of a formal space collapses at $E_{2}$. Spaces whose Eilenberg-Moore sequence collapses at $E_{2}$ will therefore be called weakly formal.

If, for some $n$,

$$
E_{2}=E_{3}=\cdots=E_{n}
$$

then the space is called weakly $n$-formal. A space which is $n$-formal in the sense of [7] is easily seen to be weakly $n$-formal. 
We can approximate weak formality in another way. Call a space spherically $n$-formal if

$$
E_{2}^{-i, *}=E_{\infty}^{-i, *}, \quad i \geqslant n+1 .
$$

Evidently

$$
\begin{aligned}
S \text { formal } & \Rightarrow S \text { weakly formal } \Rightarrow \cdots \Rightarrow S \text { spherically } l \text {-formal } \\
& \Rightarrow \cdots \Rightarrow S \text { spherically } 0 \text {-formal. }
\end{aligned}
$$

In $[7, \S 8.13]$ it is shown that spherically 0 -formal $\nRightarrow$ weakly formal $\nRightarrow$ formal. We shall give examples showing also that spherically 0 -formal $\nRightarrow$ spherically 1 -formal $\nexists$ weakly formal and conjecture that in fact spherically $l$-formal $\nRightarrow$ spherically $(l+1)$-formal. All this is in contrast with our restatement of Theorem I which reads

TheOREM II. Assume $S$ is simply connected and $\operatorname{dim} \pi_{*}(S) \otimes \mathbf{Q}<\infty$. Then $S$ is spherically 1-formal $\Leftrightarrow H(S)$ is hyperformal.

REMARK. When $S$ is a homogeneous space then spherically 1 -formal can be replaced by spherically 0 -formal in the theorem [3, Chapter 11 , Theorem IV], but this is not true more generally even if both $H(S)$ and $\pi_{*}(S) \otimes \mathbf{Q}$ have finite dimension, as is shown in $\S 3$. The corollary $S$ formal $\Leftrightarrow H(S)$ hyperformal, under the hypotheses $\operatorname{dim} H(S)<\infty, \operatorname{dim} \pi_{*}(S) \otimes \mathbf{Q}<\infty, S$ simply connected, has a short and elegant proof [2]. This result has also been established by A. Pazitnev.

A simple translation of [7, Theorem 8.12] shows that $S$ is spherically 0 -formal if and only if every primitive homology class in $H_{*}(S)$ is spherical. Slightly more subtly, we shall establish the

3.6. Proposition. $S$ is spherically 1-formal if and only if every primitive homology class in $H^{*}(S)$ and in $H^{*}\left(K_{0}, S\right)$ is spherical. ( $K_{0}$ is as in Theorem I.)

This at least suggests why Theorem II is closely related to Theorem I.

The definitions above and Theorem II extend to the categories of path-connected topological spaces $S$ and $c$-connected commutative graded differential algebras (c-connected c.g.d.a.'s) $\left(A, d_{A}\right)$ over a field $\Gamma$ of characteristic zero, with the following modifications.

(i) $\pi_{*}(S)$ must be replaced by $\pi_{\psi}^{*}(S)$ or $\pi_{\psi}^{*}\left(A, d_{A}\right)$.

(ii) Homotopy type must be suitably defined (over $\Gamma$ ).

(iii) For nonnilpotent spaces $S$ the Eilenberg-Moore sequence although convergent may not converge to $H(\Omega S)$.

The extension runs as follows:

Let $\left(A, d_{A}\right)$ be a $c$-connected c.g.d.a over $\Gamma$. (Thus $A=\Sigma_{p \geqslant 0} A^{p}$; if $a \in A^{p}$, $b \in A^{q}$ then $a b=(-1)^{p q} b a, d_{A}$ is a derivation of degree 1 and square zero, and $H^{0}\left(A, d_{A}\right)=\Gamma$.) A (minimal) model for $A$ is a c.g.d.a. homomorphism $\phi:(\wedge X, d)$ $\rightarrow\left(A, d_{A}\right)$ for which $(\wedge X, d)$ is a (minimal) $\mathrm{KS}$ complex and $\phi^{*}: H(\wedge X) \rightarrow H(A)$ is an isomorphism. If $(\wedge X, d)$ is minimal then $X^{0}=0$. (A $K S$ (Koszul-Sullivan) complex is a differential algebra of the form $(\wedge X, d)$ in which $X=\Sigma_{p>0} X^{p}$ and admits a well-ordered homogeneous basis $x_{\alpha}$ such that $d x_{\alpha}$ is a polynomial in the $x_{\beta}$ with $\beta<\alpha$. It is minimal if these polynomials have no linear term.) 
A basic result of Sullivan $[10, \S 5 ; 5]$ asserts the existence and uniqueness (up to c.g.d.a. isomorphism) of minimal models. If $(\wedge X, d)$ is a model for $\left(A, d_{A}\right)$ then $d$ induces a quotient differential $Q(d)$ in the space $Q(\wedge X)=\wedge^{+} X / \wedge^{+} X \cdot \wedge^{+} X$ of indecomposables. $Q(d)=0$ if and only if $(\wedge X, d)$ is minimal. The cohomology space $H(Q(\wedge X), Q(d))$ (which is isomorphic to $X$ if $(\wedge X, d)$ is minimal) is independent of the model and is denoted by $\pi_{\psi}^{*}\left(A, d_{A}\right)$.

Now suppose $S$ is a path-connected topological space. Then Sullivan defines a c.g.d.a. $(A(S), d)$ over $\Gamma[\mathbf{1 0}, \S 7$ or 5 , Chapter 15], natural in $S$ and whose cohomology is naturally isomorphic with $H(S ; \Gamma)$. The minimal model of $(A(S), d)$ is called the minimal model of $S$, and we write $\pi_{\psi}^{*}(A(S), d)=\pi_{\psi}^{*}(S)$.

A second basic result of Sullivan [10, Theorem $8.1 ; 1]$ asserts that if $S_{1}, S_{2}$ are simply connected with finite rational Betti numbers, and if $\Gamma=\mathbf{Q}$ then

(i) $\pi_{\psi}^{*}\left(S_{i}\right) \cong \operatorname{Hom}_{Z}\left(\pi_{*}\left(S_{i}\right)\right.$; Q), naturally in $S_{i}$, and

(ii) $S_{1}$ and $S_{2}$ have the same rational homotopy type $\Leftrightarrow$ they have isomorphic minimal models.

The definition of rational homotopy type is thus extended by

Definition. Two path-connected spaces (or $c$-connected c.g.d.a.'s over $\Gamma$ ) have the same $\Gamma$-homotopy type if their minimal models (over $\Gamma$ ) are isomorphic as c.g.d.a.'s.

The definition of formality is

Definition. A path-connected space $S$ (respectively, a $c$-connected c.g.d.a. $\left(A, d_{A}\right)$ ) is formal if $(A(S), d)$ and $(H(S), 0)$ (respectively, $\left(A, d_{A}\right)$ and $(H(A), 0)$ ) have minimal models isomorphic as c.g.d.a.'s.

The Eilenberg-Moore spectral sequence for $\left(A, d_{A}\right)$ is obtained by filtering the bar construction on $\left(A, d_{A}\right)$; details can be found in [7, §7]. The Eilenberg-Moore sequence for a path-connected space $S$ is the sequence for $(A(S), d)$. With these conventions the definitions of weakly formal and spherically $l$-formal given earlier apply verbatim and we have

TheOREM III. Let $\left(A, d_{A}\right)$ be a c-connected c.g.d.a. with $\operatorname{dim} \pi_{\psi}^{*}\left(A, d_{A}\right)<\infty$. Then $\left(A, d_{A}\right)$ is spherically 1-formal $\Leftrightarrow H(A)$ is hyperformal.

Clearly this theorem implies the identical result for path-connected spaces (replace $A$ by $A(S)$ ) and hence contains the topological Theorem II.

The proofs of the theorems rely on the filtered models of [7]. After some preliminaries in $\$ 2$, these are described in $\$ 3$ where also are the examples and the proof of Proposition 3.1. The actual proofs of the theorems are in $\$ 4$; these, however, depend on the results of $\$ 5$.

The second major ingredient in these proofs is a careful analysis of finitely generated models whose cohomology algebra is also finitely generated, and this is deferred to $\$ 5$.

As a byproduct of this analysis we obtain one final result. Let $\left(A, d_{A}\right)$ have finitely generated cohomology, and suppose $\operatorname{dim} \pi_{\psi}^{*}\left(A, d_{A}\right)<\infty$. Set

$$
\chi_{\pi}\left(A, d_{A}\right)=\operatorname{dim} \pi_{\psi}^{\text {even }}\left(A, d_{A}\right)-\operatorname{dim} \pi_{\psi}^{\text {odd }}\left(A, d_{A}\right)
$$


and

$$
f_{H(A)}(t)=\sum_{p \geqslant 0} \operatorname{dim} H^{p}(A) t^{p}
$$

Similarly, if $\wedge X$ is the minimal model we set $f_{\wedge X}(t)=\Sigma_{p \geqslant 0} \operatorname{dim}(\wedge X)^{p} t^{p}$.

Because $\operatorname{dim} X<\infty, f_{\wedge X}(t)$ is convergent for $|t|<1$. Because $H(\wedge X)=H(A)$, $\operatorname{dim}(\wedge X)^{p} \geqslant \operatorname{dim} H^{p}(A)$ and so $f_{H(A)}(t)$ is convergent for $|t|<1$. Set (following Hsiang)

$$
\rho_{0}(H(A))=\inf \left\{\alpha \mid \lim _{t \rightarrow 1-}(1-t)^{\alpha} f_{H(A)}(t)=0\right\}
$$

As is shown, for instance in [4, Proposition 2], $\rho_{0}(H(A))$ is the Krull dimension of the commutative algebra $H^{\text {even }}(A)$.

In [4, Proposition 2] it is shown that $\chi_{\pi}\left(A, d_{A}\right)-\rho_{0}(H(A)) \leqslant 0$. On the other hand in [4] is defined a fourth quadrant spectral sequence $E_{i}^{p, q}$, converging to $H(A)=H(\wedge X)$, called the odd spectral sequence. (The definition is recalled in 2.2.) An immediate consequence of Proposition 5.6 and Lemma 5.8 in this paper is

THEOREM IV. Let $A$ be a c-connected c.g.d.a. such that $\pi_{\psi}^{*}\left(A, d_{A}\right)$ is finitedimensional and $H(A)$ is a finitely generated algebra. Let $k$ be the largest integer such that (in the odd spectral sequence) $E_{\infty}^{*,-k} \neq 0$. Then

$$
k=\rho_{0}(H(A))-\chi_{\pi}\left(A, d_{A}\right) .
$$

2. Preliminaries. In this section we recall material which will be needed in the sequel. There are three distinct parts: $\wedge$-extensions, Koszul complexes, and dimension theory for commutative rings.

2.1. $\wedge$-extensions. A $\wedge$-extension is a sequence of $\mathrm{KS}$ complexes

$$
(\wedge X, d) \stackrel{i}{\rightarrow}(\wedge X \otimes \wedge Y, d) \stackrel{\rho}{\rightarrow}(\wedge Y, \bar{d})
$$

in which $i$ and $\rho$ are the obvious inclusion and projection. Note that the differential in $\wedge X \otimes \wedge Y$ need not be of the form $d \otimes 1 \pm 1 \otimes \bar{d}$. If $\phi:(\wedge X, d) \rightarrow\left(A, d_{A}\right)$ is any c.g.d.a. homomorphism between $c$-connected c.g.d.a.'s, and if $(\wedge X, d)$ is a $\mathrm{KS}$ complex then there is a commutative diagram

$$
\begin{aligned}
& \left(A, d_{A}\right) \\
& (\wedge X, d) \underset{i}{\stackrel{\phi \nearrow}{\rightarrow}}(\wedge X \otimes \wedge Y, d) \underset{\rho}{\rightarrow} \quad(\wedge Y, \bar{d})
\end{aligned}
$$

in which the bottom row is a $\wedge$-extension, $\psi^{*}$ is an isomorphism, and $(\wedge Y, \bar{d})$ is minimal-cf. [5, Theorem 6.1].

2.2. Koszul complexes. Suppose $A$ is an algebra, $a_{1}, \ldots, a_{m}$ are in the centre of $A$, and let $X$ be a space with basis $x_{1}, \ldots, x_{m}$. A differential space $(A \otimes \wedge X, d)$ is defined by $d(A)=0, d x_{i}=a_{i}$ and indeed this is the classical Koszul complex [8]. Since $d$ is homogeneous of degree -1 with respect to the grading $A \otimes \wedge X=\Sigma_{k} A \otimes$ $\wedge^{k} X$, a grading is induced in the cohomology, and we write this $H(A \otimes \wedge X)=$ $\Sigma_{k} H_{k}(A \otimes \wedge X)$. Of course if $A=\sum A^{p}$ is a c.g.a. and we set $\operatorname{deg} x_{i}=\operatorname{deg} a_{i}-1$ 
then $A \otimes \wedge X$ becomes a c.g.d.a. with bigraded cohomology $H=\Sigma H_{k}^{p}$. Note in any case that $H_{0}=A / I, I$ the ideal generated by the $a_{i}$.

Suppose now that $(\wedge Z, d)$ is a connected $\mathrm{KS}$ complex. Write $Z^{e}=Z^{\text {even }}$, $Z^{o}=Z^{\text {odd }}$ and define an associated Koszul complex $(\wedge Z, d)=\left(\wedge Z^{e} \otimes \wedge Z^{o}\right.$, $\left.d_{\sigma}\right)$ by $d_{\sigma}\left(Z^{e}\right)=0, d_{\sigma}\left(Z^{0}\right) \subset \wedge Z^{e}$ and

$$
d z-d_{\sigma} z \in \wedge Z^{e} \otimes \wedge^{+} Z^{o}, \quad z \in Z^{o} .
$$

In this case the lower gradation is given by the grading $\Sigma_{k} \wedge Z^{e} \otimes \wedge^{k} Z^{o}$, and we write $H\left(\wedge Z, d_{\sigma}\right)=\Sigma H_{k}^{p}\left(\wedge Z, d_{\sigma}\right)$.

Filter $\wedge Z$ by setting $F^{p}(\wedge Z)^{r}=\Sigma_{k \geqslant p-r}\left(\wedge Z^{e} \otimes \wedge^{k} Z^{o}\right)^{r}$. The resulting spectral sequence (introduced in [4] and called the odd spectral sequence) converges to $H(\wedge Z, d)$ and its $E_{0}, E_{1}$ and $E_{2}$ terms are given by

$$
\left(E_{0}, d_{0}\right)=\left(\wedge Z, d_{\sigma}\right) \text { and } E_{1}^{p, q}=E_{2}^{p, q}=H_{-q}^{p+q}\left(\wedge Z, d_{\sigma}\right) .
$$

Thus it is a fourth quadrant spectral sequence.

2.3. Dimension theory. Let $R$ be a noetherian integral domain over our ground field $\Gamma$. Any ideal $I \subset R$ is the finite irredundant intersection of primary ideals $Q_{j}$ whose prime ideals $P_{j}$ are called the associated prime ideals of $I$. Following [11] we write $\underline{\operatorname{dim}} P_{j}=$ transc. degree of the quotient field of $R / P_{j}$ and $\operatorname{dim}_{I}=\inf _{j} \operatorname{dim}_{j}$.

The chief result we need is a straightforward consequence of [11, Theorem 2.1, p. 195; Theorem 26, p. 203]. The result asserts that if $R$ is a polynomial algebra over $\Gamma$ on $n$ variables then $x_{1}, \ldots, x_{s}$ is a prime sequence if and only if the ideal $I$ generated by $x_{1}, \ldots, x_{s}$ satisfies $\operatorname{dim} I=n-s$. In this case $I$ is unmixed, i.e. every associated prime ideal $P$ of $I$ satisfies $\operatorname{dim} P=n-s$. As a consequence we have that any permutation of a prime sequence in $R$ is a prime sequence.

For any ideal $I \subset R$ the dimension of $I$ in $R$ depends only on $R / I$ and is called the Krull dimension of $R / I$.

Let $a_{1}, \ldots, a_{s}$ be a sequence of elements of even degree in a graded commutative algebra $A$. Let $Y$ have as basis $y_{1}, \ldots, y_{s}$ with $\operatorname{deg} y_{i}=\operatorname{deg} a_{i}-1$ and consider the Koszul complex $(A \otimes \wedge Y, d)$ with $d y_{i}=a_{i}$. Using the argument of [4, Lemma 2] it is easy to see that

2.4. Lemma. The sequence $a_{1}, \ldots, a_{s}$ is prime if and only if $H_{+}(A \otimes \wedge Y)=0$.

3. Filtered models. Let $H$ be a connected c.g.a. The minimal model $(\wedge X, d)$ of $(H, 0)$ carries an additional structure [7, §3]: $X$ has a second, lower grading $X=\Sigma_{k \geqslant 0} X_{k}$ such that, for the induced grading in $\wedge X, d$ is homogeneous of degree -1 and $H_{+}(\wedge X, d)=0, H_{0}(\wedge X, d)=H$. In particular $X_{0}$ is a minimal set of generators for $H$. The model $(\wedge X, d)$ is called the bigraded model.

Next, if $\left(A, d_{A}\right)$ is any $c$-connected c.g.d.a. the bigraded model $(\wedge X, d)$ of $H(A)$ can be perturbed to a (not necessarily minimal) model $(\wedge X, D)$ for $\left(A, d_{A}\right)$ so that $D-d: X_{k} \rightarrow \sum_{j<k-1}(\wedge X)_{j}$. This is called the filtered model for $\left(A, d_{A}\right)-\mathrm{cf}$. [7, §4] -and is minimal if and only if $\left(A, d_{A}\right)$ is weakly formal [7, Theorem 7.20].

3.1. REMARK. If $H$ is hyperformal then $X_{\geqslant 2}=0$ and so no perturbations are possible. Thus $H$ is intrinsically formal. 
Set $\bar{X}^{p, q}=X_{-p^{-1}}^{p+1}$ and extend the bigrading to $\wedge \bar{X}=\Sigma_{p \leqslant-1}(\wedge \bar{X})^{p, *}$. Identity $Q(\wedge X) \cong X=\bar{X}$ and denote the differential $Q(D)$, transported to $\bar{X}$ and extended to $\wedge \bar{X}$, by $\bar{D}$. Filtering $\wedge \bar{X}$ by the left-hand degree produces a spectral sequence which (from $E_{2}$ on) is isomorphic with the spectral sequence of Eilenberg-Moore [7, Theorem 7.14]. It follows easily that $\left(A, d_{A}\right)$ is spherically 1 -formal if and only if $\bar{X}^{-1, *} \oplus \bar{X}^{-2, *} \rightarrow H(\bar{X}, \bar{D})$ is injective. This is equivalent to the condition

$$
X_{0} \oplus X_{1} \rightarrow H(X, Q(D)) \text { is injective. }
$$

On the other hand let $(\wedge Z, d)$ be any model for $\left(A, d_{A}\right)$, and let $U \subset$ ker $d$ map isomorphically to a minimal subspace of $H^{+}(\wedge Z)$ which generates the algebra $H(\wedge Z)$. The projection $\wedge^{+} Z \rightarrow Z$ induces a map $U \rightarrow H(Z, Q(d))$ and in [7, Theorem 8.12] it is shown that either of the conditions

(3.3) $X_{0} \rightarrow H(X, Q(D))$ is injective $((\wedge X, D)$ the filtered model), or

(3.4) $U \rightarrow H(Z, Q(d))$ is injective is equivalent to spherical 0 -formality for $\left(A, d_{A}\right)$.

We come now to the examples. Because $([10, \S 8])$ a KS complex $(\wedge Z, d)$ with $Z^{1}=Z^{0}=0$ and $\operatorname{dim} Z^{p}<\infty$, all $p$, can be realized as the model of a simply connected space we need only construct the KS complex.

3.5. EXAMPLE. Our first example is a filtered model which is spherically 1 -formal but not weakly formal. In fact, the cohomology algebra $H$ is even intrinsically spherically l-formal.

Let $H$ be the algebra $\wedge\left(u_{7}, u_{7}^{\prime}, u_{9}, u_{9}^{\prime}, u_{9}^{\prime \prime}, u_{11}\right) / I$ where $I$ is generated by $u_{7} u_{7}^{\prime}, u_{7} u_{11}-u_{9} u_{9}^{\prime}, u_{7}^{\prime} u_{11}-u_{9}^{\prime} u_{9}^{\prime \prime}$, and subscripts denote degrees. If $(\wedge X, d)$ denotes the bigraded model for $(H, 0)$ then $X_{0}, X_{1}, X_{2}, X_{3}, X_{4}$ have bases

$$
\begin{gathered}
X_{0}: u_{7}, u_{7}^{\prime}, u_{9}, u_{9}^{\prime}, u_{9}^{\prime \prime}, u_{11}, \quad X_{1}: v_{13}, v_{17}, v_{17}^{\prime}, \\
X_{2}: w_{19}, w_{19}^{\prime}, w_{32}, \ldots, \quad X_{3}: z_{25}, z_{25}^{\prime}, \ldots, \quad X_{4}: y_{31}, y_{31}^{\prime}, \ldots
\end{gathered}
$$

in which the missing elements all have degrees $\geqslant 32$. Moreover $d$ is given by

$$
\begin{aligned}
& d v_{13}=u_{7} u_{7}^{\prime}, \quad d v_{17}=u_{7} u_{11}-u_{9} u_{9}^{\prime}, \quad d v_{17}^{\prime}=u_{7}^{\prime} u_{11}-u_{9}^{\prime} u_{9}^{\prime \prime}, \\
& d w_{19}=v_{13} u_{7}, \quad d w_{19}^{\prime}=v_{13} u_{7}^{\prime}, \quad d w_{32}=v_{17} u_{7} u_{9}^{\prime}, \\
& d z_{25}=w_{19} u_{7}, \quad d z_{25}^{\prime}=w_{19}^{\prime} u_{7}^{\prime} \text {, }
\end{aligned}
$$

and

$$
d y_{31}=z_{25} u_{7}, \quad d y_{31}^{\prime}=z_{25}^{\prime} u_{7}^{\prime} .
$$

Now define a perturbation $D$ by setting

$$
\begin{aligned}
& D=d \text { in } X_{0}, X_{1}, \quad D w_{19}=v_{13} u_{7}-u_{9}^{\prime} u_{11}, \quad D w_{19}^{\prime}=d w_{19}^{\prime}, \\
& D z_{25}=w_{19} u_{7}-v_{17} u_{9}^{\prime}, \quad D z_{25}^{\prime}=d z_{25}^{\prime}, \quad D y_{31}=z_{25} u_{7}-w_{32} .
\end{aligned}
$$

Because $H^{p}=0, p \geqslant 30$, this operator extends to a differential $D$ in $\wedge X$ such that $(D-d): X_{k} \rightarrow \Sigma_{j<k-1}(\wedge X)_{j}$. Note that $Q(D) y_{31}=w_{32}$.

The resulting filtered model is trivially even intrinsically spherically 1-formal, but not minimal. Hence it is not weakly formal. 
3.5'. EXAMPLE. We construct a spherically 0 -formal model which satisfies $\operatorname{dim} \pi_{\psi}^{*}$ $<\infty, \operatorname{dim} H^{*}<\infty$ but is not spherically 1-formal. This shows that the hypothesis of spherically l-formal in Theorem III is essential, and also that spherically 0 -formal $\nRightarrow$ spherically 1 -formal.

Define a minimal $\mathrm{KS}$ complex $(\wedge Z, d)$ as follows. $Z$ has as basis $u_{3}, v_{3}, w_{5}, a_{4}, b_{4}, x_{7}, y_{7}$ (subscripts denote degrees) and $d u=d v=d a=d b=0$, $d w=u v, \quad d x=u w-a^{2}, d y=v w-b^{2}$. The spherical cohomology is then $[u],[v],[a],[b]$. A straightforward computation shows that a basis of the cohomology is given by $1,[u],[v],[a],[b],[a][u],[a][v],[b][u],[b][v],[a][b],[a]^{2},[b]^{2}$, $[a][b][u],[a][b][v],[b]^{2}[u],[a]^{3},[a]^{2}[b],[a][b]^{2},[b]^{3},[a][b]^{2}[u],[b]^{3}[u],[a]^{3}[b]$, $[a][b]^{3}$ and $[a][b]^{3}[u]$. Thus $U=(u, v, a, b)$ generates $H(\wedge Z)$ so that $(\wedge Z, d)$ is spherically 0 -formal by (3.4). It is clearly not hyperformal and so, by Theorem III, not spherically 1 -formal.

We establish next the geometric characterization of spherical 1-formality.

3.6. Proposition. A simply connected space $S$ with finite rational Betti numbers is spherically 1-formal if and only if the primitive homology classes in $H_{*}(S)$ and $H_{*}\left(K_{0}, S\right)$ are all spherical $\left(K_{0}\right.$ as in the introduction).

Proof. Because a generating space for $H^{*}(S)$ is dual to the primitive subspace of $H_{*}(S)$, we may assume that the map $\phi_{0}: S \rightarrow K_{0}$ of the introduction is represented by the inclusion $\wedge X_{0} \rightarrow(\wedge X, D)$ in the filtered model.

Passing to cohomology we obtain the short exact sequence

$$
0 \rightarrow H^{*}\left(K_{0}, S\right) \rightarrow \wedge X_{0} \rightarrow H(\wedge X, D)=H(S) \rightarrow 0 .
$$

Since $D=d$ in $X_{1}$, and since $H(\wedge X, D)=H(\wedge X, d)=\wedge X_{0} \wedge \wedge X_{0} \cdot d\left(X_{1}\right)$, we can identify $H^{*}\left(K_{0}, S\right)$ with the ideal $\wedge X_{0} \cdot d\left(X_{1}\right)$ in $\wedge X_{0}$ as a $\wedge X_{0}$-algebra. It follows at once that $d\left(X_{1}\right)$ is dual to the primitive subspace of $H_{*}\left(K_{0}, S\right)$. It is now easy to see that the inclusion

$$
\left(\wedge X_{0} \otimes \wedge X_{1}, d\right) \rightarrow(\wedge X, D)
$$

represents $\phi_{1}: S \rightarrow E$ of the introduction.

In the diagram

$$
\begin{aligned}
& \pi_{*}(S) \otimes \mathbf{Q} \stackrel{\left(\phi_{0}\right)_{\sharp}}{\rightarrow} \pi_{*}\left(K_{0}\right) \otimes \mathbf{Q} \quad \rightarrow \quad \pi_{*}\left(K_{0}, S\right) \otimes \mathbf{Q} \stackrel{\stackrel{\partial}{\rightarrow}}{\rightarrow} \pi_{*}(S) \otimes \mathbf{Q} \\
& \downarrow h_{1} \quad \downarrow h_{2} \quad \downarrow h_{3} \\
& P_{*}(S) \underset{\left(\phi_{0}\right)_{*}}{\stackrel{\cong}{\rightarrow}} \quad P_{*}\left(K_{0}\right) \quad \rightarrow \quad P_{*}\left(K_{0}, S\right)
\end{aligned}
$$

(in which the $h_{i}$ are the Hurewicz homomorphisms) note that $\operatorname{Im} h_{2} \subset$ primitive subspace $\subset \operatorname{Im}\left(\phi_{0}\right)_{*}$. Hence $h_{3}$ factors over $\partial$ to yield a linear map $f: \operatorname{ker}\left(\phi_{0}\right)_{\sharp} \rightarrow$ $P_{*}\left(K_{0}, S\right)$.

Since $\left(\phi_{0}\right)_{\sharp}$ is dual to the map $X_{0} \rightarrow H(X, Q(D))$ it follows (by a straightforward check) that $f$ is dual to the composite

$$
g: X_{1} \rightarrow H(X, Q(D)) \rightarrow H(X, Q(D)) / \operatorname{Im} X_{0} .
$$


To say that every primitive class is spherical in $H_{*}\left(K_{0}, S\right)$ is to say that $f$ is surjective. This is equivalent to $g$ injective. Similarly the primitive classes in $H_{*}(S)$ are all spherical if and only if $X_{0} \rightarrow H(X, Q(D))$ is injective.

In view of (3.2) this completes the proof. Q.E.D.

4. The main theorems. Preliminary to the proof of Theorems I and III we use filtered models to put the minimal model of spherically l-formal space in a desirable form.

Let $(\wedge X, D) \stackrel{m}{\rightarrow}\left(A, d_{A}\right)$ be the filtered model of a $c$-connected c.g.d.a. Apply 2.1 to the inclusion $\left(\wedge X_{0} \otimes \wedge X_{1}, d\right) \rightarrow(\wedge X, D)$ to obtain a c.g.d.a. homomorphism $\psi:\left(\wedge X_{0} \otimes \wedge X_{1} \otimes \wedge T, d\right) \rightarrow(\wedge X, D)$ such that $\psi^{*}$ is an isomorphism and $(\wedge T, \bar{d})$ is minimal.

\subsection{Proposition. If $\left(A, d_{A}\right)$ is spherically 1-formal then}

$$
m \circ \psi:\left(\wedge X_{0} \otimes \wedge X_{1} \otimes \wedge T, d\right) \rightarrow\left(A, d_{A}\right)
$$

is the minimal model.

Proof. This is clearly a model. Since $(\wedge T, \bar{d})$ and $\left(\wedge X_{0} \otimes \wedge X_{1}, d\right)$ are minimal we have (identifying $X_{0} \oplus X_{1} \oplus T=Q\left(\wedge X_{0} \otimes \wedge X_{1} \otimes \wedge T\right)$ ) that $T^{Q(d)} \rightarrow X_{0} \oplus X_{1} \stackrel{Q(d)}{\rightarrow} 0$. Since $\psi^{*}$ is an isomorphism the linear part of $\psi$ defines an isomorphism $Q(\psi)^{*}: H\left(X_{0} \oplus X_{1} \oplus T, Q(d)\right) \stackrel{\cong}{\rightarrow} H(X, Q(D))$-cf. [5, Theorem 7.2].

Now spherical 1-formality shows that $X_{0} \oplus X_{1} \rightarrow H\left(X_{0} \oplus X_{1} \oplus T, Q(d)\right)$ is injective; hence $Q(d)=0$ and the model is minimal. Q.E.D.

The main result is Theorem 4.2 below. Combined with its corollary and Proposition 3.6 (including the remark in the proof of Proposition 3.6 that $\left(\wedge X_{0} \otimes \wedge X_{1}\right.$, d) $\rightarrow(\wedge X, D)$ represents $\left.\phi_{1}\right)$ it immediately implies both Theorems I and III.

4.2. THEOREM. Assume that $\left(A, d_{A}\right)$ is spherically 1-formal, c-connected, and that $\operatorname{dim} \pi_{\psi}^{*}\left(A, d_{A}\right)<\infty$. Then the filtered model for $\left(A, d_{A}\right)$ has the form $(\wedge X, d)$ with $X=X_{0} \oplus X_{1}, X_{1}=X_{1}^{\text {odd }}$, and $X_{j}=0, j \geqslant 2$.

4.3. CoROllary. If $x_{1}, \ldots, x_{m}$ is a basis for $X_{1}$ then $d x_{1}, \ldots, d x_{m}$ is a prime sequence in $\wedge X_{0}$.

Proof. Apply Lemma 2.4, noting that necessarily $H_{+}\left(\wedge X_{0} \otimes \wedge X_{1}, d\right)=0$. Q.E.D.

4.4. Proof of Theorem 4.2. The minimal model for $\left(A, d_{A}\right)$ has the form of Proposition 4.1, and hence $\operatorname{dim} X_{0}, \operatorname{dim} X_{1}$ and $\operatorname{dim} T$ are all finite. Since $H(A)=$ $\wedge X_{0} / \wedge X_{0} \cdot d\left(X_{1}\right)$ it is a finitely generated algebra.

Now write $Y=X_{0}^{\text {even }}, W=X_{0}^{\text {odd }} \oplus X_{1} \oplus T, Z=X_{0} \oplus X_{1} \oplus T$ so that $\left(\wedge X_{0} \otimes\right.$ $\left.\wedge X_{1} \otimes \wedge T, d\right)=(\wedge Z, d)=(\wedge Y \otimes \wedge W, d)$ exhibits the minimal model of $\left(A, d_{A}\right)$ as a $\wedge$-extension. If $y_{1}, \ldots, y_{l}$ is a basis for $Y$ define $\bar{Y}$ to be a graded space with basis $\bar{y}_{i}$, and set $\operatorname{deg} \bar{y}_{i}=2 \operatorname{deg} y_{i}-1$. Extend $d$ to $\wedge Z \otimes \wedge \bar{Y}$ by putting $d \bar{y}_{i}=y_{i}^{2}$. 
The construction of $(\wedge X, D)$ is such that every cohomology class is represented by an element in $\wedge X_{0}$. This is therefore also true in $\wedge Z$. Hence the argument at the end of [4, Proposition 2] shows that $H(\wedge Z \otimes \wedge \bar{Y}, d)$ has finite dimension. But by $[6$, Corollary 5.13] this implies that

$$
H(\wedge W) \otimes \wedge \bar{Y}=H(\wedge W \otimes \wedge \bar{Y}, \bar{d})
$$

has finite dimension, where $\bar{d}$ denotes the differential induced by putting $Y=0$. Thus the $\wedge$-extension $\wedge Y \otimes \wedge W$ satisfies the conditions in Proposition 5.1(ii) below.

We now apply the results in $\S 5$. In particular we can find $c_{1}, \ldots, c_{r} \in \wedge Y$ satisfying the conditions of Lemmas 5.2 and 5.7. As in Lemma 5.7 let $U$ be a graded space with basis $u_{1}, \ldots, u_{r}$ and degree $u_{i}=$ degree $c_{i}-1$. Extend $(\wedge Z, d)$ to $(\wedge Z \otimes \wedge U, D)$ by putting $D u_{i}=c_{i}$.

Lemma 5.7 asserts that $\operatorname{dim} H(\wedge Z \otimes \wedge U, D)<\infty$. If $n$ is the top degree such that $H^{n}(\wedge Z \otimes \wedge U, D) \neq 0$, then Lemma 5.8 asserts that

$$
\lambda^{*}: H^{n}(\wedge Z, d) \rightarrow H^{n}(\wedge Z \otimes \wedge U, D)
$$

is surjective.

On the other hand write

$$
\wedge Z \otimes \wedge U=\left(\wedge X_{0} \otimes \wedge X_{1}^{\text {odd }} \otimes \wedge U\right) \otimes\left(\wedge X_{1}^{\text {even }} \otimes \wedge T\right) .
$$

This also exhibits $(\wedge Z \otimes \wedge U, D)$ as a $\wedge$-extension. By the proof of Proposition 5.6, $H\left(\wedge Z, d_{\sigma}\right)$ is finitely generated as a module over $\wedge\left(c_{1}, \ldots, c_{r}\right)$. Hence so is $H(\wedge Z, d)$, because the odd spectral sequence converges from $H\left(\wedge Z, d_{\sigma}\right)$ to $H(\wedge Z, d)$.

Let $I, J$, and $K \subset \wedge X_{0}$ be the ideals generated by $d\left(X_{1}\right), d\left(X_{1}^{\text {odd }}\right)$ and $d\left(X_{1}^{\text {odd }}\right)+$ $\left(c_{1}, \ldots, c_{r}\right)$. Then $H(\wedge Z, d)=H(A)=\wedge X_{0} / I$ and so $\wedge X_{0} / I$ is a finitely generated $\wedge\left(c_{1}, \ldots, c_{r}\right)$ module. Since $I$ is generated by $J$, together with finitely many elements of odd degree, $\wedge X_{0} / J$ is also a finitely generated $\wedge\left(c_{1}, \ldots, c_{r}\right)$ module. Hence $\operatorname{dim} \wedge X_{0} / K<\infty$ and it follows that

$$
\operatorname{dim} H\left(\wedge X_{0} \otimes \wedge X_{1}^{\text {odd }} \otimes \wedge U\right)<\infty .
$$

On the other hand, since $(\wedge Z \otimes \wedge U, D)$ is minimal, [6, Corollary 5.13] shows that $\operatorname{dim} H\left(\wedge X_{1}^{\text {even }} \otimes \wedge T, \bar{D}\right)<\infty$. If we apply [4, Theorem 3] to each of $\left(\wedge X_{0} \otimes\right.$ $\left.\wedge X_{1}^{\text {odd }} \otimes \wedge U, D\right),\left(\wedge X_{1}^{\text {even }} \otimes \wedge T, \bar{D}\right)$ and $(\wedge Z \otimes \wedge U, D)$, we find that the top degrees $n_{1}, n_{2}, n$ in which the cohomology is nonzero satisfy $n=n_{1}+n_{2}$.

But we know from above that $H^{n}(\wedge Z) \rightarrow H^{n}(\wedge Z \otimes \wedge U)$ is nonzero. Since every cohomology class in $H(\wedge Z)$ can be represented by an element of $\wedge X_{0}$, it follows that $H^{n}\left(\wedge X_{0} \otimes \wedge X_{1}^{\text {odd }} \otimes \wedge U\right) \rightarrow H^{n}(\wedge Z \otimes \wedge U)$ is nonzero. Hence $n_{1}$ $\geqslant n$ and so $n_{2}=0$.

We now have that $H^{+}\left(\wedge X_{1}^{\text {even }} \otimes \wedge T, \bar{D}\right)=0$. Since $\left(\wedge X_{1}^{\text {even }} \otimes \wedge T, \bar{D}\right)$ is minimal we conclude that $X_{1}^{\text {even }}=T=0$, and hence that the minimal model for $\left(A, d_{A}\right)$ has the form $\left(\wedge X_{0} \otimes \wedge X_{1}, d\right)$ with $X_{1}^{\text {even }}=0$. Q.E.D. 
5. Finitely generated models with finitely generated cohomology. Let $\left(A, d_{A}\right)$ be a c-connected c.g.d.a.

5.1. Proposition. The following two conditions on $\left(A, d_{A}\right)$ are equivalent.

(i) $\operatorname{dim} \pi_{\psi}^{*}\left(A, d_{A}\right)<\infty$ and $H(A)$ is a finitely generated algebra.

(ii) There is a model for $\left(A, d_{A}\right)$ of the form $(\wedge Z, d)=\wedge Y \otimes \wedge W$ in which $(\wedge Y, 0) \rightarrow(\wedge Z, d) \stackrel{\rho}{\rightarrow}(\wedge W, \bar{d})$ is a $\wedge$-extension, $\operatorname{dim} H(\wedge W, \bar{d}), \operatorname{dim} Y$ and $\operatorname{dim} W$ are finite and $Y$ is evenly graded.

Proof. (ii) $\Rightarrow$ (i) Filter $\wedge Z$ using the degree of $\wedge Y$ to get a spectral sequence converging to $H(\wedge Z)$ with $E_{2}$-term $\wedge Y \otimes H(\wedge W)$. It follows that $E_{2}$ (hence also $E_{\infty}$ and $H(\wedge Z)$ ) are finitely generated $\wedge Y$ modules. Thus $H(\wedge Z)$ is a finitely generated algebra.

(i) $\Rightarrow$ (ii) Let $(\wedge X, d)$ be the minimal model of $\left(A, d_{A}\right)$ and let $Y$ be an evenly graded space of finite dimension such that there is a homomorphism $\psi:(\wedge Y, 0) \rightarrow$ $(\wedge X, d)$ which makes $H(\wedge X)$ into a finitely generated $\wedge Y$ module. Use 2.1 to produce a $\wedge$-extension $(\wedge Y, 0) \rightarrow(\wedge Z, d) \stackrel{\rho}{\rightarrow}(\wedge W, \bar{d})$ and an extension of $\psi$ to a homomorphism $\phi:(\wedge Z, d) \rightarrow(\wedge X, d)$ such that $\phi^{*}$ is an isomorphism. Do this so that $(\wedge W, \bar{d})$ is minimal.

Identify $Z=Y \oplus W$ and $X$ with the respective indecomposable spaces for $\wedge Z, \wedge X$ and note [5, Theorem 7.2] that $\phi$ induces an isomorphism $Q(\phi)^{*}$ : $H(Y \oplus W, Q(d)) \stackrel{\cong}{\rightarrow} X$. Because $Q(\bar{d})=0, Q(d): W \rightarrow Y$ and it follows that $\operatorname{dim} W$ $<\infty$. It remains to show that $\operatorname{dim} H(\wedge . W, \bar{d})<\infty$.

Define a graded space $\bar{Y}$ by $\bar{Y}^{p}=Y^{p+1}$ and extend $(\wedge Z, d)$ to $(\wedge Z \otimes \wedge \bar{Y}$, d) by putting $d \bar{y}=y$ where $\bar{y}$ corresponds to $y$ under the identification $\bar{Y}=Y$. Use the grading $\Sigma_{k} \wedge Z \otimes \wedge^{k} \bar{Y}$ to obtain a spectral sequence whose $E_{1}$ term is the Koszul complex $(H(\wedge Z) \otimes \wedge \bar{Y}, D)$ with $D \bar{y}=[y]$. Since $H(\wedge Z)$ is a finitely generated $\wedge Y$ module, $H_{0}(H(\wedge Z) \otimes \wedge \bar{Y})$ has finite dimension. But $H(H(\wedge Z) \otimes \wedge \bar{Y})$ is finitely generated as an $H_{0}(H(\wedge Z) \otimes \wedge \bar{Y})$ module, and it follows that $H(H(\wedge Z) \otimes \wedge \bar{Y})$ has finite dimension. This implies $\operatorname{dim} H(\wedge W, \bar{d})$ $=\operatorname{dim} H(\wedge Z \otimes \wedge \bar{Y}, d)<\infty$. Q.E.D.

Now consider a $\wedge$-extension $\wedge Z=Y \otimes \wedge W$ satisfying the conditions of Proposition 5.1. Let $\rho:(\wedge Z, d) \rightarrow(\wedge W, \bar{d})$ be the projection and write $W^{o}=W^{\text {odd }}$, $W^{e}=W^{\text {even }}$.

5.2. Lemma. Let $N$ be an integer divisible by the degrees of the homogeneous elements in $Y$. There is then a prime sequence in $\wedge Y \otimes \wedge W^{e}$ of the form

$$
a_{1}, \ldots, a_{s}, b_{1}, \ldots, b_{t}, c_{1}, \ldots, c_{r},
$$

and satisfying the following conditions.

(i) $a_{i}=d_{\sigma} v_{i}, b_{j}=d_{\sigma} v_{j+s}$ for (not necessarily homogeneous) elements $v_{l} \in W^{o}$; and for any $w \in W^{o}, a_{1}, \ldots, a_{s}, b_{1}, \ldots, b_{t}, d_{\sigma} w$ is not prime.

(ii) $\bar{d}_{\sigma} v_{i}(1 \leqslant i \leqslant s)$ is a prime sequence in $\wedge W^{e}$.

(iii) $c_{k} \in(\wedge Y)^{N}, 1 \leqslant k \leqslant r$.

(iv) $s=\operatorname{dim} W^{e}$ and $r+t=\operatorname{dim} Y$. 
5.4. Remark. The ideal $J$ generated by the $a_{i}, b_{j}, c_{k}$ satisfies $\operatorname{dim} J=0$ (cf. 2.3) and so $\operatorname{dim} \wedge Z^{e} / J<\infty$.

Proof of Lemma 5.2. By [4, Proposition 1], $\operatorname{dim} H\left(\wedge W, \bar{d}_{\sigma}\right)<\infty$, and so [4, Lemma 8] yields $v_{1}, \ldots, v_{s} \in W^{o}$ with $s=\operatorname{dim} W^{e}$ so that $\bar{d}_{o} v_{i}$ is a prime sequence in $\wedge W^{e}$. Then a basis of $Y$, followed by $d_{\sigma} v_{1}, \ldots, d_{\sigma} v_{s}$, is a prime sequence in $\wedge Z^{e}$. Since a permutation of a prime sequence is prime, $d_{\sigma} v_{1}, \ldots, d_{\sigma} v_{s}$ is a prime sequence in $\wedge Z^{e}$.

Extend this to a maximal prime sequence in $\wedge Z^{e}$ of the form $d_{\sigma} v_{1}, \ldots, d_{\sigma} v_{s}$, $d_{\sigma} v_{s+1}, \ldots, d_{\sigma} v_{s+t}$ with $v_{l} \in W^{o}$. Extend this in turn to a maximal prime sequence in $\wedge Z^{e}$ of the form $d_{\sigma} v_{1}, \ldots, d_{\sigma} v_{s+t}, c_{1}, \ldots, c_{r}$, with $c_{k} \in(\wedge Y)^{N}$.

The argument of $\left[4\right.$, Lemma 8] shows that $(\wedge Y)^{N}$ is contained in one of the prime ideals $P$ associated with the ideal $J$ generated by this sequence. Since $(\wedge Y)^{N}$ contains a power of every homogeneous element of $Y$ we conclude that $\wedge^{+} Y \subset P$ and hence $\operatorname{ker} \rho \subset P$.

Moreover $\bar{d}_{\sigma} v_{1}, \ldots, \bar{d}_{\mathrm{o}} v_{s} \in \rho(P)$ and hence $\rho(P)$ is a prime ideal of $\operatorname{dim} 0$ in $\wedge W^{e}$. It follows that $\operatorname{dim} P=0$ and so, by $\S 2.3, s+t+r=\operatorname{dim} Z^{e}$. Q.E.D.

5.5. LemMA. With the hypotheses and notation of Lemma 5.2 let $k$ be the largest integer such that $H_{k}\left(\wedge Z, d_{\sigma}\right) \neq 0$. Then $k=\operatorname{dim} W^{o}-s-t$.

Proof. Let $I=\cap_{j} Q_{j}$ be the noetherian decomposition of the ideal $I$ generated by $a_{1}, \ldots, b_{t}$ in $\wedge Z^{e}$ and let $P_{j}$ be the prime ideal associated with the primary ideal $Q_{j}$. The maximality of $a_{1}, \ldots, b_{t}$ means that $d_{o} w$ is in some $P_{j}$ for each $w \in W^{o}$. Hence by the argument of [4, Lemma 8], $d_{\sigma}\left(W^{o}\right) \subset P_{1}$ say. Choose $q_{j} \in Q_{j}$ so that $q_{j} \notin P_{1}$ (possible because $I$ is unmixed so that $P_{i} \not \subset P_{1}$ for any $i>1$ ). Set $q=\Pi_{j} q_{j}$.

Now $q \notin P_{1}$ and so $q \notin I$. Since some power of any $d_{\sigma} w$ is in $Q_{1}$, that power multiplied by $q$ is in $I$. By multiplying $q$ by suitable powers of the $d_{\sigma} w$ we find an element $\Phi \in \wedge Z^{e}$ such that $\Phi \notin I$ but such that $\left(d_{\sigma} w\right) \Phi \in I, w \in W^{o}$.

Choose now homogeneous elements $w_{1}, \ldots, w_{k} \in W^{o}$ which together with $v_{1}, \ldots, v_{s+t}$ give a basis. Thus $k=\operatorname{dim} W^{o}-s-t$. A projection

$$
\pi:\left(\wedge Z, d_{\sigma}\right) \rightarrow\left(\wedge Z^{e} / I \otimes \wedge\left(w_{1}, \ldots, w_{k}\right), D\right)
$$

is given by the obvious projection in $\wedge Z^{e}$ together with $\pi\left(v_{l}\right)=0, \pi\left(w_{i}\right)=w_{i}$. Because the $d_{\sigma} v_{l}$ are a prime sequence $\pi^{*}$ is an isomorphism of cohomology, homogeneous of lower degree zero.

In particular $H_{p}\left(\wedge Z, d_{\sigma}\right)=0, p>k$. Moreover by construction $\left(D w_{i}\right) \pi \Phi=0$ $(1 \leqslant i \leqslant k)$ and $\pi \Phi \neq 0$. Thus $\pi \Phi \otimes w_{1} \wedge \cdots \wedge w_{k}$ is a nonzero cocycle (and hence represents a nonzero class) in $H_{k}\left(\wedge Z^{e} / I \otimes \wedge\left(w_{1}, \ldots, w_{k}\right)\right)$. Thus $H_{k}\left(\wedge Z, d_{\sigma}\right) \neq 0$. Q.E.D.

5.6. Proposition. With the notation and hypotheses above let $k$ be the maximum integer such that $H_{k}\left(\wedge Z, d_{\sigma}\right) \neq 0$. Then

$$
\rho_{0}(H(\wedge Z, d))=\rho_{0}\left(H\left(\wedge Z, d_{\sigma}\right)\right)=k+\chi_{\pi}(\wedge Z) .
$$


Proof. Since the odd spectral sequence converges from $H\left(\wedge Z, d_{\sigma}\right)$ we have $\rho_{0}(H(\wedge Z, d)) \leqslant \rho_{0}\left(H\left(\wedge Z, d_{\sigma}\right)\right)$. Next we claim that the inclusion $\wedge\left(c_{1}, \ldots, c_{r}\right) \rightarrow$ $\wedge Z^{e}$ induces inclusions $\wedge\left(c_{1}, \ldots, c_{r}\right) \rightarrow H\left(\wedge Z, d_{\sigma}\right)$ and $\wedge\left(c_{1}, \ldots, c_{r}\right) \rightarrow$ $H(\wedge Z, d)$.

Indeed, recall from the proof of Lemma 5.5 that one of the prime ideals $P_{1}$ for the ideal generated by $a_{1}, \ldots, b_{t}$ contains $d_{\sigma}\left(W^{o}\right)$. The argument of [4, Proposition 2] shows that the map $\wedge\left(c_{1}, \ldots, c_{r}\right) \rightarrow \wedge Z^{e} / P_{1}$ is an inclusion. Hence so is $\wedge\left(c_{1}, \ldots, c_{r}\right) \rightarrow \wedge Z^{e} /\left(d_{\sigma} W^{o}\right)=H_{0}\left(\wedge Z, d_{o}\right)$.

Next, suppose that for some $\Phi \in \wedge\left(c_{1}, \ldots, c_{r}\right), \Phi=d \Psi, \Psi \in \wedge Z$. Write $\Psi=$ $\Psi_{0}+\cdots+\Psi_{m}, \Psi_{i} \in \wedge Z^{e} \otimes \wedge^{i} W^{o}$. Then the component of $d \Psi$ in $\wedge Z^{e}$ is $d_{\sigma} \Psi_{1}$ so that $\Phi=d_{\sigma} \Psi_{1}$. This implies that $\Phi=0$ by the above argument, so that the second map is also an inclusion. From this we deduce that $r \leqslant \rho_{0}(H(\wedge Z, d))$.

On the other hand, by the remark after Lemma 5.2, $\wedge Z^{e}$ is finitely generated as a module over $\wedge\left(a_{1}, \ldots, a_{s}, b_{1}, \ldots, b_{t}, c_{1}, \ldots, c_{r}\right)$. Hence $H_{0}\left(\wedge Z, d_{\sigma}\right)$ is finitely generated over $\wedge\left(c_{1}, \ldots, c_{r}\right)$. Thus $H\left(\wedge Z, d_{\sigma}\right)$ is finitely generated as a module over $\wedge\left(c_{1}, \ldots, c_{r}\right)$, and so $\rho_{0}\left(H\left(\wedge Z, d_{\sigma}\right)\right) \leqslant r$. The various inequalities we have derived give

$$
\rho_{0}(H(\wedge Z, d))=\rho_{0}\left(H\left(\wedge Z, d_{\sigma}\right)\right)=r .
$$

Finally (using Lemma 5.5) $\chi_{\pi}(\wedge Z)+k=\operatorname{dim} Z^{e}-\operatorname{dim} W^{o}+\operatorname{dim} W^{o}-s-t$ $=(s+r+t)-s-t=r$. Q.E.D.

Let $\wedge Z$ be as in the previous lemmata. Choose the integer $N$ of Lemma 5.2 so that $H_{k}^{p}\left(\wedge Z, d_{\sigma}\right) \neq 0$ for some $p<N$. Choose a graded space $U$ with basis $u_{1}, \ldots, u_{r}$ and degree $u_{i}=N-1$; thus $U=U^{\text {odd }}$. Define a KS complex $(\wedge Z \otimes$ $\wedge U, D$ ) by putting $D=d$ in $\wedge Z$ and $D u_{i}=c_{i}$ (chosen as in Lemma 5.2). Then clearly $D_{\sigma}=d_{\sigma}$ in $\wedge Z$ and $D_{\sigma} u_{i}=c_{i}$.

5.7. LEMMA. With the hypotheses and notation above

(i) $H(\wedge Z \otimes \wedge U, D)$ and $H\left(\wedge Z \otimes \wedge U, D_{\sigma}\right)$ have finite dimension.

(ii) $H_{l}\left(\wedge Z \otimes \wedge U, D_{o}\right)=0, l>k$, where $k=\operatorname{dim} W^{o}-s-t$.

(iii) The map $H_{k}\left(\wedge Z, d_{\sigma}\right) \rightarrow H_{k}\left(\wedge Z \otimes \wedge U, D_{\sigma}\right)$ is nonzero.

Proof. The remark after Lemma 5.2 shows that $\operatorname{dim} H_{0}\left(\wedge Z \otimes \wedge U, D_{\sigma}\right)<\infty$ and (i) follows. Let $J$ be the ideal generated by $a_{1}, \ldots, c_{r}$. As in Lemma 5.5 we have a projection

$$
\left(\wedge Z \otimes \wedge U, D_{\sigma}\right) \rightarrow\left(\wedge Z^{e} / J \otimes \wedge\left(w_{1}, \ldots, w_{k}\right)\right)
$$

which induces a cohomology isomorphism, and (ii) follows.

Finally $\wedge Z \rightarrow \wedge Z \otimes \wedge U$ is an isomorphism in degrees $\leqslant N-2$ and injective in degree $N-1$. Thus $H^{p}\left(\wedge Z, d_{\sigma}\right) \rightarrow H^{p}\left(\wedge Z \otimes \wedge U, D_{\sigma}\right)$ is injective for $p \leqslant N-1$. In particular $H_{k}^{p}\left(\wedge Z, d_{\sigma}\right) \rightarrow H_{k}^{p}(\wedge Z \otimes \wedge U)$ is nonzero for some $p$. Q.E.D.

Let $(\wedge Z \otimes \wedge U, D)$ be as in the previous lemma. Because $\operatorname{dim} Z \oplus U<\infty$ and $\operatorname{dim} H(\wedge Z \otimes \wedge U, D)<\infty$ we can apply the results of $[4, \S 8]$. These assert that $H(\wedge Z \otimes \wedge U, D)$ and $H\left(\wedge Z \otimes \wedge U, D_{\sigma}\right)$ are Poincare duality algebras with fundamental classes of the same degree, say $n$. Moreover if $k$ is as in Lemma 5.7 then $H^{n}\left(\wedge Z \otimes \wedge U, D_{\sigma}\right)=H_{k}^{n}\left(\wedge Z \otimes \wedge U, D_{\sigma}\right)$. 
Consider now the inclusion $\lambda:(\wedge Z, d) \rightarrow(\wedge Z \otimes \wedge U, D)$. It induces a homomorphism of odd spectral sequences $\lambda_{i}:\left(E_{i}, d_{i}\right) \rightarrow\left(\check{E}_{i}, \check{d}_{i}\right)$ with

$$
\lambda_{0}=\lambda:\left(\wedge Z, d_{\sigma}\right) \rightarrow\left(\wedge Z \otimes \wedge U, D_{\sigma}\right)
$$

and

$$
\lambda_{1}=\lambda_{2}=\lambda_{0}^{*}: H\left(\wedge Z, d_{\sigma}\right) \rightarrow H\left(\wedge Z \otimes \wedge U, D_{\sigma}\right) .
$$

5.8. LEMMA. With the hypotheses and notation above the maps

(i) $\left(\lambda_{0}^{*}\right)^{n}: H_{k}^{n}\left(\wedge Z, d_{\sigma}\right) \rightarrow H_{k}^{n}\left(\wedge Z \otimes \wedge U, D_{\sigma}\right)$,

(ii) $\lambda_{\infty}^{n+k,-k}: E_{\infty}^{n+k,-k} \rightarrow \check{E}_{\infty}^{n+k,-k}$, and

(iii) $\lambda^{*}: H^{n}(\wedge Z, d) \rightarrow H^{n}(\wedge Z \otimes \wedge U, D)$

are surjective.

Proof. (i) Because $\operatorname{dim} H_{k}^{n}\left(\wedge Z \otimes \wedge U, D_{\sigma}\right)=1$ we need only show that $\left(\lambda_{0}^{*}\right)^{n}$ is nonzero. By Lemma 5.7 we can (for some $p$ ) find $\alpha \in H_{k}^{p}\left(\wedge Z, d_{\sigma}\right)$ with $\lambda_{0}^{*}(\alpha) \neq 0$. Since $k$ is the top nonzero lower degree for $H\left(\wedge Z \otimes \wedge U, D_{\sigma}\right)$, Poincaré duality gives an element $\beta \in H_{0}^{n-p}\left(\wedge Z \otimes \wedge U, D_{\sigma}\right)$ such that $\left(\lambda_{0}^{*} \alpha\right) \cdot \beta \neq 0$.

But $\beta$ is represented by some $\Phi \in\left(\wedge Z^{e}\right)^{n-p}$. Let $\gamma$ be the class in $H_{0}^{n-p}\left(\wedge Z, d_{\sigma}\right)$ represented by $\Phi$. Then clearly $\lambda_{0}^{*}(\gamma)=\beta$ and so $\lambda_{0}^{*}(\alpha \cdot \gamma)=\left(\lambda_{0}^{*} \alpha\right) \cdot \beta \neq 0$.

(ii) and (iii) Choose a class $\varepsilon$ in $H_{k}^{n}\left(\wedge Z, d_{\sigma}\right)$ such that $\lambda_{0}^{*} \varepsilon \neq 0$. Because $H_{i}\left(\wedge Z, d_{\sigma}\right)=0, i>k$, a simple spectral sequence argument shows that $\varepsilon$ survives to $\bar{\varepsilon} \in E_{\infty}^{n+k,-k}$. Clearly $\lambda_{0}^{*} \varepsilon$ survives to $\lambda_{\infty}^{n+k,-k}(\bar{\varepsilon})$; hence by [4, Theorem 3] the latter class is nonzero. Q.E.D.

\section{REFERENCES}

1. A. K. Bousfield and V. K. A. M. Gugenheim, On PL de Rham theory and rational homotopy type, Mem. Amer. Math. Soc. No. 179 (1976).

2. Y. Felix, Thèse, Universite de Louvain la Neuve, Belgique.

3. W. Greub et al., Connections, curvature, and cohomology. Vol. III, Academic Press, New York, 1975.

4. S. Halperin, Finiteness in the minimal model of Sullivan, Trans. Amer. Math. Soc. 230 (1977), 173-199.

5. 1977.

6. Lectures on minimal models, Publ. Internes de l'U.E.R. de Math. de Université de Lille I, Rational fibrations, minimal models and fibrings of homogeneous spaces, Trans. Amer. Math. Soc. 244 (1978), 199-223.

7. S. Halperin and J. D. Stasheff, Obstructions to homotopy equivalences, Adv. in Math. 32 (1979), 233-279.

8. J.-L. Koszul, Sur un type d'algèbres différentielles en rapport aver la transgression, Colloque de Topologie (Espaces Fibrés) (Bruxelles, 1950), Thone, Liège; Masson, Paris, 1951, pp. 73-81.

9. L. Smith, Homological algebra and the Eilenberg-Moore spectral sequence, Trans. Amer. Math. Soc. 129 (1967), 58-93.

10. D. Sullivan, Infinitesimal computations in topology, Inst. Hautes Études Sci. Publ. Math. 47 (1978), 269-331.

11. O. Zariski and P. Samuel, Commutative algebra. Vol. II, Van Nostrand, Princeton, N. J., 1960.

Institut de Mathematiques Pures, Universite de Louvain la Neuve, Louvain-la-Neuve, BELGIQUE

Physical Sciences Division, Scarborough College, University of Toronto, Toronto, Ontario, CANADA 